\title{
RNA-directed DNA Methylation and Pol IVb in Arabidopsis
}

\author{
M. Matzke, T. Kanno, B. Huettel, L. Daxinger, and A.J.M. Matzke \\ Gregor Mendel Institute of Molecular Plant Biology, Austrian Academy of Sciences, A-1030 Vienna, Austria
}

\begin{abstract}
Recent work in Arabidopsis has revealed a plant-specific RNA polymerase, pol IV, that is specialized for RNA interference (RNAi)-mediated, chromatin-based gene silencing. Two functionally diversified pol IV complexes have been identified: pol IVa is required to produce or amplify the small RNA trigger, whereas pol IVb, together with the plant-specific SWI/SNF-like chromatin remodeling factor DRD1, acts downstream from small RNA formation to induce de novo cytosine methylation of homologous DNA by an unknown mechanism. Retrotransposon long terminal repeats (LTRs) and other unannotated sequences that encode small RNAs are prime targets for DRD1/pol IVb-mediated cytosine methylation. In $d r d l$ and pol IVb mutants, silent LTRs in euchromatin can be derepressed, resulting in enhanced transcription of adjacent genes or intergenic regions. In addition to mediating de novo methylation, some evidence suggests that DRD1 and pol IVb are also involved in a reciprocal process of active demethylation, perhaps in conjunction with DNA glycosylase domain-containing proteins such as ROS1. We speculate that DRD1/pol IV-dependent methylation/demethylation evolved in the plant kingdom as a means to facilitate rapid, reversible changes in gene expression, which might have adaptive significance for immobile plants growing in unpredictable environments.
\end{abstract}

Several RNAi-mediated pathways operate in the nucleus to induce epigenetic modifications in a sequencespecific manner (Matzke and Birchler 2005). In a wide range of organisms, including fission yeast, Drosophila, and plants, the RNAi machinery is used to package tandem repeats, such as those in pericentromeric regions, into heterochromatin containing histone $\mathrm{H} 3$ lysine 9 (H3K9) methylation (Bernstein and Allis 2005; Kavi et al. 2005). In principle, RNAi-mediated heterochromatin assembly can occur without detectable DNA cytosine methylation, which is absent in fission yeast and adult flies. It is known, however, that RNA can guide methylation of cytosines in DNA as illustrated by the phenomenon of RNA-directed DNA methylation, which has been documented most thoroughly in plants. Despite initial reports of RNA-directed DNA methylation in human cells, recent data suggest that this process requires several plant-specific proteins - most notably, an unusual RNA polymerase IV - which has strengthened the possibility that RNA-directed DNA methylation is limited to the plant kingdom.

In this paper, we discuss genetic screens that have identified proteins needed for RNA-directed DNA methylation and transcriptional gene silencing in Arabidopsis thaliana, and we describe features of endogenous DNA target sequences that are methylated and silenced by these proteins. We propose a hypothetical mechanism for RNAdirected DNA methylation and speculate on a potential connection with the reciprocal process of active demethylation of 5-methylcytosine. After discussing related RNAi-mediated transcriptional gene silencing pathways in other organisms, we consider the possible importance of this silencing pathway for plants.

\section{DISCOVERY AND CHARACTERISTICS OF RNA-DIRECTED DNA METHYLATION}

Evidence for a sequence-specific signal for DNA methylation was initially obtained in transgenic plants containing multiple, unlinked copies of identical promoters, which became methylated and transcriptionally silenced in a reversible, homology-dependent manner (Matzke et al. 1989; Vaucheret 1993; Park et al. 1996). Although it was initially unclear whether DNA-DNA or RNA-DNA interactions were involved in the homologous sequence interactions that led to methylation and silencing, the discovery of RNA-directed DNA methylation in viroid-infected tobacco plants shifted the experimental focus to the latter possibility (Wassenegger et al. 1994). Further work on promoter-dependent silencing demonstrated that double-stranded RNAs containing promoter sequences could direct methylation and transcriptional silencing of homologous promoters in trans (Jones et al. 1999, 2001; Mette et al. 1999, 2000; Aufsatz et al. 2002a). Processing of the double-stranded RNAs to small RNAs 21-24 nucleotides in length suggested a mechanistic link to RNAi (Mette et al. 2000; Sijen et al. 2001).

Detailed analysis of sequences modified by RNAdirected DNA methylation in plants revealed two distinctive features of the modification pattern: First, methylation is largely restricted to the region of RNA-DNA sequence homology and second, methylation is acquired at cytosines in all sequence contexts $(\mathrm{CG}$, $\mathrm{CNG}$, and $\mathrm{CNN}$, where $\mathrm{N}$ is $\mathrm{A}, \mathrm{T}$, or $\mathrm{C}$ ) (Pélissier et al. 1999; Pélissier and Wassenegger 2000). This complex pattern renders DNA methylation more versatile in plants than in most other organisms that methylate their DNA. For example, plant sequences that are deficient in CG dinucleotides, which are the exclusive sites of methylation in mammals, but rich in asymmetrical CNN nucleotide groups can be methylated and potentially silenced by RNA-directed DNA methylation. In addition, there is a difference in heritability of cytosine methylation during DNA replication depending on the sequence context: Whereas symmetrical CG methylation can be maintained through the action of maintenance methyltransferases that act on hemimethylated substrates, asymmetrical CNN 
methylation is not efficiently maintained and needs the continuous presence of the inducing RNA (Jones et al. 2001; Aufsatz et al. 2002a). Thus, CNN methylation can be rapidly lost in dividing cells if the RNA trigger is withdrawn. Because it is poorly maintained, CNN methylation can be regarded as a measure of de novo methylation in the presence of RNA. As discussed below, the facile reversibility of CNN methylation has implications for plant promoters that are silenced by $\mathrm{CNN}$ methylation but unaffected by CG methylation.

\section{PROTEINS REQUIRED FOR RNA-DIRECTED DNA METHYLATION}

Forward and reverse genetics approaches in Arabidopsis have revealed that RNA-directed DNA methylation requires for the most part conserved DNA methyltransferases and histone modifying enzymes (Mathieu and Bender 2004; Chan et al. 2005). The DRM type of DNA methyltransferase catalyzes de novo methylation of cytosines in all sequence contexts within a region of RNA-DNA sequence homology (Cao et al. 2003). In addition, however, full de novo methylation of $\mathrm{CG}$ dinucleotides requires the CG-specific methyltransferase MET1 (Aufsatz et al. 2004), which might act at the de novo step in a manner similar to that of its postreplicative maintenance function on hemimethylated DNA (Fig. 1). DRM, which stands for domains rearranged methyltransferase, is homologous to the Dnmt3 group in mammals. As the name implies, however, the catalytic domains are in a different order in plants (VI, IX, X, I-V) than in mammals (I-VI, IX, X) (Cao et al. 2000). The different arrangement might extend the substrate specificity of DRM enzymes beyond CG dinucleotides, thus accounting for the extensive $\mathrm{CNG}$ and $\mathrm{CNN}$ methylation observed in plant nuclear DNA. Maintenance of symmetrical CG and CNG methylation during DNA replication is executed, respectively, by MET1, which is homologous to mammalian Dnmt1, and the plant-specific CHROMOMETHYLASE3 (CMT3) (Fig. 1) (Mathieu and Bender 2004; Chan et al. 2005).
Forward genetic screens have identified two histonemodifying enzymes that participate in the RNA-directed DNA methylation pathway. HDA6, which is one of 18 histone deacetylases encoded in the Arabidopsis genome, is needed to maintain and reinforce $\mathrm{CG}$ methylation induced by RNA (Aufsatz et al. 2002b). SUVH4/KYP is a H3K9 methyltransferase that contributes to maintenance of CNG methylation (Jackson et al. 2002; Malagnac et al. 2002).

RNA-directed DNA methylation requires at least three proteins of the RNAi machinery to generate and use small RNAs that guide epigenetic modifications and silencing. RDR2, one of six RNA-dependent RNA polymerases encoded in the Arabidopsis genome, is thought to synthesize double-stranded RNA from single-stranded primary transcripts; DCL3, one of four dicer-like proteins in Arabidopsis, processes double-stranded RNA to the heterochromatin-specific 24-nucleotide small RNAs; and AGO4, one of ten argonaute proteins in Arabidopsis, presumably binds small RNAs in nuclear effector complexes that facilitate DNA and histone methylation (Xie et al. 2004; Chan et al. 2005).

\section{DISCOVERY OF DRD1 AND POL IVB}

The identification of RNAi proteins and enzymes that catalyze epigenetic modifications in response to RNA signals provides information about components of the RNA-directed DNA methylation pathway but does not illuminate how RNA accesses the homologous target DNA to provide a substrate for de novo cytosine methylation. We have recently completed a mutant screen that has the potential to provide insight into this process. The screen exploited a transgene system based on the seedspecific $\alpha^{\prime}$ promoter, which was used to drive expression of a target gene encoding green fluorescent protein (GFP). A second, unlinked "silencer" complex encodes a hairpin RNA comprising $\alpha$ ' promoter sequences. In wildtype plants, the hairpin RNA is processed to small RNAs, which trigger methylation and silencing of the homologous target $\alpha^{\prime}$ promoter, resulting in GFP-negative seeds.

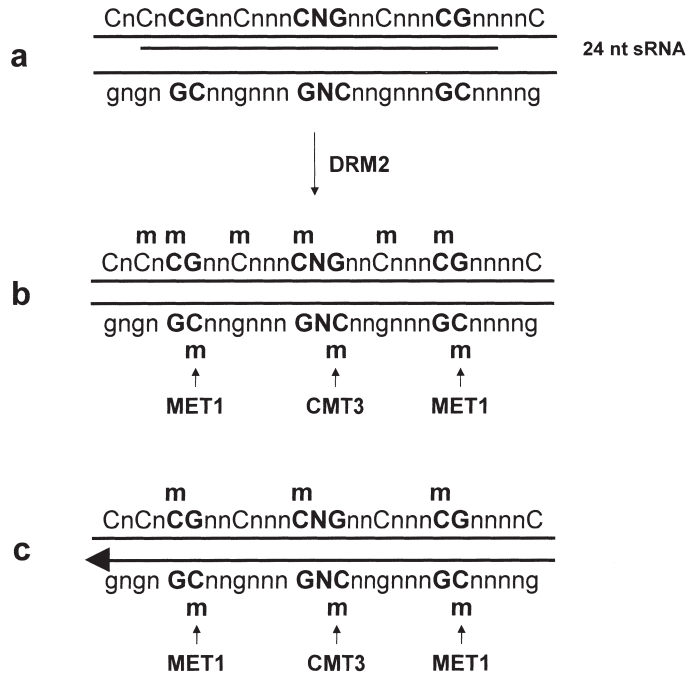

Figure 1. DNA methyltransferases cooperate to establish and maintain RNA-directed DNA methylation: a hypothetical model. (a) A 24nucleotide small RNA base-pairs to the complementary DNA strand (top), providing a signal for DRM2 to catalyze de novo methylation (m) of cytosines in all sequence contexts within the region of RNA-DNA sequence complementarity. (b) The resulting hemimethylated CGs and CNGs (methylated on one strand but not the other) are recognized by MET1 and CMT3, respectively, which catalyze methylation of the opposite cytosines on the bottom DNA strand. These steps, which are not directly guided by RNA, can be regarded as "de novo" methylation because they occur on unmethylated single strands in nonreplicating DNA. The small RNA-targeted DNA is now fully methylated at CGs and CNGs, whereas CNN methylation is still limited to the top (RNAtargeted) strand. (c) During rounds of DNA replication (leftward arrow), methylation at symmetrical CGs and CNGs will be maintained by MET1 and CMT3, respectively, because they recognize the hemimethylated substrate (methylated on the parental strand, unmethylated on the daughter strand). In contrast, asymmetrical CNN methylation is eventually lost during rounds of DNA replication in the absence of the RNA trigger. 
In retrospect, the choice of the $\alpha^{\prime}$ promoter as a target promoter was fortunate, because it turned out to be an excellent reporter of silencing by $\mathrm{CNN}$ methylation, which, as mentioned above, is indicative of RNA-directed de novo methylation. Moreover, the factors we identified in the mutant screen using this promoter turned out to be expressed throughout the plant, not only in seeds.

Following chemical mutagenesis of seeds doubly homozygous for the target and silencer complexes, mutants were identified by examining M2 seeds (representing the first generation in which a recessive mutation can be homozygous) for recovery of GFP activity. The screen retrieved three complementation groups, named $d r d$ for defective in RNA-directed DNA methylation. Subsequent map-based cloning revealed that DRD1 is a plant-specific SWI/SNF-like, putative chromatin remodeling protein (Kanno et al. 2004). DRD2 and DRD3 are subunits of a novel RNA polymerase, pol IV, which is found only in plants (Kanno et al. 2005b).

In the three $d r d$ mutants, the target $\alpha^{\prime}$ promoter displayed an unusual pattern of methylation in which most CG methylation was retained, whereas non-CG methylation was lost. This pattern indicated that the $\alpha^{\prime}$ promoter is not silenced by CG methylation but primarily by non$\mathrm{CG}$ methylation. In particular, the heavy loss of $\mathrm{CNN}$ methylation in the mutants suggested defects in de novo methylation (Kanno et al. 2004). This hypothesis was tested in experiments designed to assess de novo and maintenance methylation in the $d r d 1$ mutant. (In the context of RNA-directed DNA methylation, de novo methylation is defined as methylation that is induced on unmethylated DNA upon introduction of the RNA trigger; maintenance methylation is defined as the methylation that is retained following removal of the RNA trigger.) These experiments indeed showed that DRD1 is required for RNA-directed de novo methylation of target promoters. Surprisingly, however, experiments using the "maintenance setup" revealed that DRD1 is also required for full loss of CG methylation after segregating the silencer complex that is the source of the inducing RNA (Kanno et al. 2005a). These results led to the proposal that DRD1 facilitates dynamic regulation of DNA methylation: This putative chromatin remodeling factor is needed to direct methylation in response to RNA signals, but it is also required to fully erase methylation when the trigger RNA is withdrawn. We presume that the same applies to DRD2 and DRD3, because the methylation patterns are identical in all three $d r d$ mutants (Kanno et al. 2004, 2005b).

DRD2 and DRD3 correspond, respectively, to the second largest and largest subunits of pol IV, the existence of which was first inferred from the analysis of the Arabidopsis genome sequence (Arabidopsis Genome Initiative 2000). Accordingly, DRD2 and DRD3 have been renamed NRPD2a and NRPD1b, respectively. NRPD2a appears to be the only expressed second largest subunit of pol IV in Arabidopsis (Onodera et al. 2005). In contrast, NRPD1b has a homolog, NRPD1a, which was first identified as SDE4 in a forward genetic screen for silencing defective mutants (Dalmay et al. 2000; Herr et al. 2005). NRPD2a teams up with each of the two distinct largest subunits to generate two functionally diversified pol IV complexes. Whereas both pol IV complexes are ultimately required for methylation of target DNA, they act at different steps in the pathway. pol IVa, containing NRPD1a and NRPD2a, is needed to produce or amplify the small RNA trigger. In contrast, pol IVb, containing NRPD1b and NRPD2a, acts downstream from this step to somehow use small RNA signals to guide cytosine methylation of the homologous DNA region by an unknown mechanism. Indeed, it is still unclear whether either pol IV complex transcribes to any significant extent and if so whether the template is DNA or RNA (Herr 2005; Vaucheret 2005; Vaughn and Martienssen 2005). In a later section, we present a speculative model for how the two pol IV complexes might cooperate to establish and maintain RNA-directed DNA methylation.

The distinct roles of NRPD1a and NRPD1b in RNAdirected DNA methylation can probably be attributed to differences in their carboxy-terminal domains (CTDs), which is the most divergent region between the two proteins. NRPD $1 b$ has an extended CTD that contains an additional 522 amino acids that are not present in NRPD1a. The possible function of the extended CTD in NRPD1b can be considered by comparing it to the CTD of the largest subunit of RNA polymerase II (pol II), which transcribes mRNA precursors. The CTD of pol II is unique among subunits of DNA-dependent RNA polymerase in consisting solely of different numbers of copies of a heptapeptide repeat, Tyr-Ser-Pro-Thr-Ser-Pro-Ser, in which the serine residues provide potential phosphorylation sites. The pol II CTD is dispensable for RNA polymerization and appears to serve as a recruitment platform for proteins that process mRNA (Buratowski 2003; Meinhart et al. 2005). However, it is increasingly appreciated that the CTD can also recruit proteins that catalyze epigenetic modifications. For example, in budding yeast, sequential phosphorylation of two serines leads to sequential recruitment of two histone methyltransferases, Set1 and Set2, which catalyze histone modifications characteristic of active transcription (methylation at $\mathrm{H} 3 \mathrm{~K} 4$ and H3K36) (Hampsey and Reinberg 2003). In human cells, many different proteins have been proposed to associate in a functional manner with the pol II CTD, including DNA methyltransferases (Carty and Greenleaf 2002), although there is not yet any information on these potential affiliations in plants.

Returning to the pol IV largest subunits: The extended CTD of NRPD1b, the largest subunit of pol IVb, does not consist solely of copies of a heptapeptide repeat, but it does contain ten copies of an imperfect 16-amino-acid repeat, which contains potential phosphorylation sites (Pontier et al. 2005). Intriguingly, the region containing these repeats is missing in NRPD1a (Fig. 2). It is tempting to speculate that the extended CTD of NRPD1b can recruit DNA methyltransferases, thus accounting for its involvement in RNA-directed de novo methylation. Determining whether this is the case will require further biochemical work and identification of proteins that interact with the CTD of NRPD1b.

Although only NRPD1b, and not NRPD1a, has an extended CTD containing repeats, both pol IV largest subunits have a motif found in DCL proteins (in this context, DCL stands for defective chloroplasts and leaves, 


\section{NRPD1b/DRD3}

(largest subunit Pol IVb)

1976 amino acids

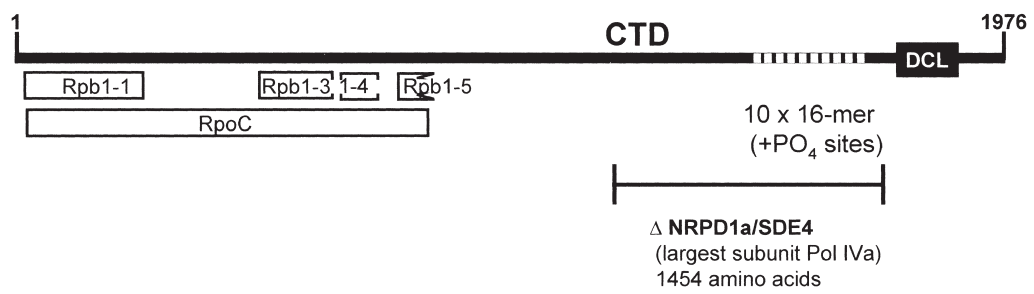

Figure 2. Largest subunits of pol IV differ in their carboxy-terminal domains. The largest subunit of pol IVb (NRPD1b/DRD3) is 1976 amino acids; the largest subunit of pol IVa (NRPD1a/SDE4) is 1454 amino acids. The size difference is due to an additional 522 amino acids in the carboxy-terminal domain (CTD) of NRPD1b. The extended CTD of NRPD1b contains ten copies of an imperfect 16amino-acid repeat (white dashes) that has potential phosphorylation sites (Pontier et al. 2005). This region may provide a recruitment platform for enzymes that induce epigenetic modifications. Both NRPD1a and NRPD1b contain a motif of unknown function that is also found in proteins called defective chloroplasts and leaves (DCL). Conserved domains that might be required for RNA polymerase activity in the amino-terminal half of NRPD1b are boxed (Kanno et al. 2005b). For a discussion of differences in the active sites of pol IV subunits as compared to their counterparts in other DNA-dependent RNA polymerases, see Herr (2005).

not dicer-like) (Fig. 2). DCL proteins are found only in plants and are thought to be involved in rRNA processing (Bellaoui and Gruissem 2004; Lahmy et al. 2004). The function of the DCL motif in the two pol IV largest subunits is presently unknown, but it might be involved in mediating interactions with RNA.

\section{ENDOGENOUS TARGETS OF DRD1 AND POL IVB}

The forward genetic screens that identified DRD1, NRPD1b, and NRPD2a (Kanno et al. 2004, 2005b) as well as NPRD1a (Dalmay et al. 2000; Herr et al. 2005) were carried out using transgene systems. Although transgenes supplied convenient readouts to monitor release of gene silencing, they did not provide information about the endogenous targets of these silencing proteins in the Arabidopsis genome. Our initial studies on possible targets of DRD1-dependent silencing and methylation focused on sequences that are reactivated or lose methylation in DDM1 (DECREASE IN DNA METHYLATION), which is also a SWI/SNF-like protein (Jeddeloh et al. 1999); however, unlike the plant-specific DRD1, DDM1 has a mammalian homolog, Lsh (Dennis et al. 2001). DNA methylation analysis of several types of tandem repeats in Arabidopsis, such as 180-bp centromeric repeats, 5S rDNA repeats, and Athila retrotransposon arrays, supported the idea that DRD1, NRPD1b, and NRPD2a (the two subunits are collectively referred to hereafter as pol $\mathrm{IVb}$ ) are important for CNN methylation, which is greatly reduced in $d r d 1$ and pol IVb mutants. In contrast, predominantly CG methylation is lost from these repeated sequences in $d d m 1$ mutants. Thus, unlike DDM1, DRD1 and pol IVb do not appear to have a decisive role in maintaining $\mathrm{CG}$ methylation in tandem repeats that are packaged into heterochromatin.

To investigate the endogenous targets of the DRD1/pol IVb-dependent silencing pathway in more detail, we carried out two approaches that identified transcripts that differentially accumulate in wild-type and mutant plants: cDNA-AFLP and suppression subtractive hybridization
(SSH). These techniques do not discriminate among protein-coding and non-protein-coding regions of the genome and can be used to detect relatively modest changes in expression. The initial experiments were carried out using a $d r d 1$ mutant. As starting material, we used polyadenylated RNA, which is enriched in pol II transcripts, isolated from seedlings.

Transcripts representing sequences that are up- or down-regulated in the $d r d 1$ mutant compared to wild-type plants were recovered from both the cDNA-AFLP and SSH approaches. Putative targets included annotated genes and unannotated intergenic regions, many of which are present in euchromatic chromosome arms or at the periphery of pericentromeric heterochromatin. Notably, tandem repeats and transposon sequences were underrepresented in the two screens. Real-time quantitative reverse transcriptase-polymerase chain reaction (RTPCR) was used to verify differences in expression levels of the candidate target genes in the $d r d l$ mutant and in other mutants in the pol IV pathway (nrpd2a, nrpd1b, $n r p d 1 a, r d r 2$ ) as well as in a met1 mutant. The validated targets could be grouped into three categories (Huettel et al. 2006; B. Huettel, unpubl.): (1) Sequences that are upregulated in a $d r d 1$ mutant and in pol IV mutants; these could be placed into three subcategories depending on whether reactivation was less, more, or approximately the same in a met 1 mutant; (2) sequences that are down-regulated in a $d r d 1$ mutant and in pol IV mutants; and (3) sequences that are up-regulated in a $d r d 1$ mutant but not consistently up-regulated in pol IV mutants. Here, we further discuss categories 1 and 2.

\section{Category 1}

Most of the candidate target sequences we identified by cDNA-AFLP and SSH analyses were up-regulated in the drd1 mutant compared to wild-type plants. Strikingly, we identified in both approaches a pair of putative targets that are adjacent to each other in the same orientation on chromosome 5: a truncated LINE element and a gene encoding the ribosomal protein RPL18C. In addition to being 
up-regulated in a $d r d 1$ mutant, these two transcripts were also found to be up-regulated in other mutants defective in the pol IV pathway (nrpdla, nrpd1b, nrpd2a, and $r d r 2$ ). Analysis of the intergenic region between LINE (long interspersed nuclear element) and the RPL18C gene revealed a previously unannotated solo LTR, which is a member of a small Copia-like retrotransposon family that we named LTRCO. The expression data suggested that the solo LTR was derepressed in the $d r d 1$ mutant, leading to bidirectional transcriptional up-regulation of the flanking sequences to produce an antisense LINE transcript and a sense $R P L 18 C$ transcript (Huettel et al. 2006).

Derepression of the solo LTR was accompanied by loss of cytosine methylation, particularly asymmetrical CNN methylation, from this element. An examination of histone modifications by chromatin immunoprecipitation (ChIP) analysis demonstrated that the solo LTR was associated with euchromatic histone modifications-H3K4 trimethylation $(\mathrm{H} 3 \mathrm{~K} 4 \mathrm{me} 3)$ and $\mathrm{H} 3$ acetylation (acetyl H3) - as well as H3K27 monomethylation (H3K27me) as the main repressive histone modification. Negligible $\mathrm{H} 3 \mathrm{~K} 9$ dimethylation (H3K9me2), which is a hallmark of constitutive heterochromatin in Arabidopsis (Fuchs et al. 2006), was detected. A similar histone modification pattern comprising a combination of euchromatic marks and H3K27me but little or no H3K9me2 was observed for the transgene $\alpha^{\prime}$ promoter. Notably, other members of the LTRCO family were not detectably reactivated in the drdl mutant. This may have been due to the fact that they were modified by $\mathrm{H} 3 \mathrm{~K} 9 \mathrm{me} 2$ and lacked euchromatic marks. The LTRs that remained silent in the $d r d 1$ mutant nevertheless lost CNN methylation in mutant plants. This result is significant because it demonstrates that even though the LTRs are not derepressed in a $d r d l$ mutant, they are targets of DRD1-dependent cytosine methylation (Huettel et al. 2006).

Consistent with the solo LTR being a target of DRD1/pol IVb-dependent silencing and methylation, 24nucleotide small RNAs originating from this sequence could be detected on northern blots. The LTR small RNAs were present at wild-type levels in $d r d 1$ and nrpd1b mutants, but absent in nrpd2a, nrpdla, and $r d r 2$ plants. This supports the view that pol IVa (containing nrpdla and nrpd2a) is involved in the production or amplification of small RNAs, whereas DRD1 and pol IVb (containing $n r p d 1 b$ and $n r p d 2 a$ ) act downstream from this step to induce DNA cytosine methylation.

We extended the analysis by examining three intergenic targets - IGI, IG2, and $I G 5$ - that are up-regulated in the $d r d 1$ and pol IVb mutants (Huettel et al. 2006). IG2 and $I G 5$ are adjacent to LTRs that encode small RNAs, whereas $I G 1$ is part of an unannotated intergenic region that is apparently bidirectionally transcribed because it encodes 19 small RNAs of both sense and antisense polarities (Lu et al. 2005). For the most part, the up-regulated $I G 1, I G 2$, and $I G 5$ sequences have patterns of histone modification that conform to those observed for the solo LTR and transgene $\alpha^{\prime}$ promoter; that is, euchromatic marks and $\mathrm{H} 3 \mathrm{~K} 27 \mathrm{me}$ but little or no H3K9me2. Only IG5 had in addition detectable H3K9me2. Strikingly, however, the $I G 5$ sequence was considerably more up-regu- lated in a metl mutant than in the $d r d 1$ and pol IVb mutants. This finding suggests that $\mathrm{CG}$ methylation is more important than CNN methylation for silencing IG5 transcription. In contrast, the solo LTR of the LTRCO family and $I G 1$ were not appreciably reactivated in the met1 mutant. IG2 represented an intermediate case that was reactivated to approximately the same degree in both the $d r d 1$ and met 1 mutants (Huettel et al. 2006). Although the basis of the variable reactivation of different targets in $d r d 1$ or met 1 mutants is not known, one possibility is that the sequence composition, particularly the number and density of $\mathrm{CG}$ dinucleotides, might have a role. Sequences that are rich in CGs and depleted in CNN nucleotide groups might reactivate more effectively in met 1 mutants than in $d r d 1$ mutants, whereas the converse may be true for sequences rich in $\mathrm{CNNs}$ and deficient in CG dinucleotides.

The presence of histone modifications typical of euchromatin at many DRD1 target genes is in agreement with their location in gene-rich chromosome arms or at the boundary between euchromatin and pericentromeric heterochromatin (Huettel et al. 2006). In contrast, the LTRs of the LTRCO family that failed to reactivate in the drdl mutant are embedded in transposon-rich pericentromeric heterochromatin. These findings substantiate the proposal that DRD1 and pol IVb act to reversibly silence promoters in euchromatin.

Although the screens we performed to identify sequences reactivated in the $d r d l$ mutant were not exhaustive, the consistency of the available data allows us to draw provisional conclusions about the characteristics of sequences that are methylated and silenced by DRD1 and pol IVb. It is likely that all sequences with homologous small RNAs can be targets of DRD1/pol IVb-mediated cytosine methylation, which is characterized by methylation of cytosines in all sequence contexts. Importantly, however, only a subset of sequences targeted for methylation is reactivated in $d r d 1$ and pol $\mathrm{IVb}$ mutants. Strongly reactivated targets tend to have a euchromatic character, reside in gene-rich regions, and be more sensitive to $\mathrm{CNN}$ methylation than to CG methylation. Further experiments using Arabidopsis wholegenome tiling arrays, which have recently become commercially available, will build on these studies of DRD1 and pol IVb target sequences.

\section{LTRs as Preferential Targets for DRD1/pol IVb-mediated Methylation}

Our investigation suggested that retrotransposon LTRs are frequent targets of DRD1/pol IVb-mediated cytosine methylation. Consistent with this, many LTRs in the $A$. thaliana genome encode small RNAs ( $\mathrm{Lu}$ et al. 2005), which could guide DRD1/pol IVb-mediated methylation of cognate DNA sequences. The targeting of LTRs by a small RNA-mediated pathway in Arabidopsis contrasts with the situation in fission yeast. Despite an early report that LTR activity in fission yeast can be regulated by RNAi-mediated chromatin modifications and influence the expression of adjacent meiotic genes (Schramke and Allshire 2003), most of the 262 LTRs in this organism do not seem to be 
targets of RNAi-mediated chromatin modifications, nor do they encode small RNAs (Cam et al. 2005).

Given that our initial mutant screen scored for reactivation of a silent transgene promoter, it is not too surprising that the natural targets of the DRD1/pol IVb silencing pathway include LTRs, which are promoter/enhancer elements that can be used by pol II. The identification of the solo LTR of the LTRCO family as a derepressed regulatory element in both the SSH and cDNA-AFLP analyses suggests that this sequence might have special features which render it particularly conspicuous to the DRD1/pol $\mathrm{IVb}$ protein machinery. Two considerations are its length and sequence composition. Like the transgene $\alpha^{\prime}$ promoter, the solo LTR is several hundred base pairs in length and deficient in CG dinucleotides, having only two CGs in 376 bp. The low CG density in these sequences might favor silencing by CNN methylation, which is the repressive mark lost most consistently in $d r d l$ and pol IVb mutants. The relatively short length of the solo LTR might prevent stable maintenance of constitutive heterochromatin (Tran et al. 2005).

A third noteworthy feature of the solo LTR is the presence of an internal tandem repeat comprising four copies of a 36-bp monomer, which is the source of a short RNA identified by massively parallel signature sequencing ( $\mathrm{Lu}$ et al. 2005). Because tandem repeats can ensure the continuous production of double-stranded RNAs and small RNAs by RNA-dependent RNA polymerase and Dicer, respectively (Martienssen 2003), they might provide a strong nucleation site for RNAi-mediated chromatin modifications. However, the correlation between tandem repeats in LTRs and the ability to be silenced by the DRD1/pol IVb machinery is not perfect. For example, although the Copia-like LTR driving transcription of IG5 contains tandem repeats, the truncated Athila LTR upstream of $I G 2$ lacks such repeats, although direct repeats are present in full-length copies of the Athila LTR present elsewhere in the genome (Huettel et al. 2006; B. Huettel, unpubl.). Moreover, as illustrated by the repeatfree transgene $\alpha^{\prime}$ promoter, tandem repeats are not essential for DRD1/pol IVb-mediated DNA methylation when silencing is induced by a hairpin RNA that is independent of RNA-dependent RNA polymerase activity (Kanno et al. 2005b). Nevertheless, many LTRs in the Arabidopsis genome contain short internal tandem repeats (Table 1), so it is worth considering in future studies whether these reiterated regions increase the probability that RNAimediated chromatin modifications will be targeted to LTRs. We were unable to detect comparable internal tandem repeats in LTRs of fission yeast (B. Huettel, unpubl.), which might at least partially explain the resistance of these elements to RNAi-mediated chromatin modifications.

\section{Category 2}

The second category of putative DRD1 targets identified in the cDNA-AFLP and SSH analyses comprises sequences that are down-regulated in the $d r d 1$ mutant. The most intriguing candidate from this category, which was subsequently validated by real-time RT-PCR, is
ROS1 (REPRESSOR OF SILENCING). ROS1 is a large, plant-specific protein that contains a DNA glycosylase domain. ROS1, and a related protein DEMETER, are thought to be involved in active demethylation by participating in a base-repair-type mechanism that removes 5-methylcytosine and replaces it with an unmethylated cytosine (Kapoor et al. 2005; MoralesRuiz et al. 2006). The down-regulation of ROS1 in the $d r d 1$ mutant and pol IVb mutants might explain the failure to fully erase CG methylation from target promoters in these mutants (Kanno et al. 2005a,b). The observation that ROS1 is down-regulated to an even greater extent in nrpdla and $r d r 2$ mutants strengthens the connection of ROS1 to the entire pol IV pathway (Huettel et al. 2006). Although the nature of this connection is not yet clear, we speculate that a DRD1/pol IVb complex can act with DNA methyltransferases to induce de novo methylation and with DNA glycosylases to remove methylation, thus keeping euchromatic promoters in a potentially reversible epigenetic state (Huettel et al. 2006). In addition to passive loss of methylation (which results from a failure to maintain methylation, in particular $\mathrm{CNN}$ methylation, during rounds of DNA replication in the absence of the RNA trigger), active demethylation through an enzymatic activity might provide an alternate way to lose cytosine methylation in the pol IV pathway. Importantly, active demethylation can occur on nonreplicating DNA, which permits epigenetic gene silencing to be reversed in stationary cells. The ability to switch gene expression states in nondividing cells by means of reversible pol-IV-mediated DNA methylation can potentially contribute to physiological adaptation in mature plant organs.

\section{POSSIBLE MECHANISM OF DRD1/POL IV- DEPENDENT DNA METHYLATION}

Our data on DRD1 and pol IVb targets suggest that this silencing pathway can reversibly silence promoters in euchromatin via cycles of de novo DNA methylation and either passive or active loss of methylation (Fig. 3). This conclusion is consistent with other proposals which suggest that pol $\mathrm{IVb}$ is recruited by euchromatic modifications left by another euchromatic RNA polymerase (Pontier et al. 2005) and that pol IV is important for genes that alternate between decondensed euchromatic states and condensed chromocenter-associated heterochromatic states (Onodera et al. 2005). Although there is general agreement about these basic features of DRD1/pol IVbdependent silencing, the mechanism by which these proteins elicit de novo methylation is unknown. Here, we present a speculative model that takes into account the unique contribution of each pol IV complex.

The DRD1/pol IVb pathway appears to be particularly important for silencing transposon promoters, such as solo LTRs, in euchromatin. Silencing prevents pol II from using these promoters to initiate transcription, which could extend into flanking plant genes and have potentially deleterious consequences, such as unscheduled synthesis of antisense RNAs. To silence transposon promoters via the pol IV pathway, an essential first step is 
Table 1. Tandem Repeats in the LTR Promoter Regions of $A$. thaliana Retrotransposons

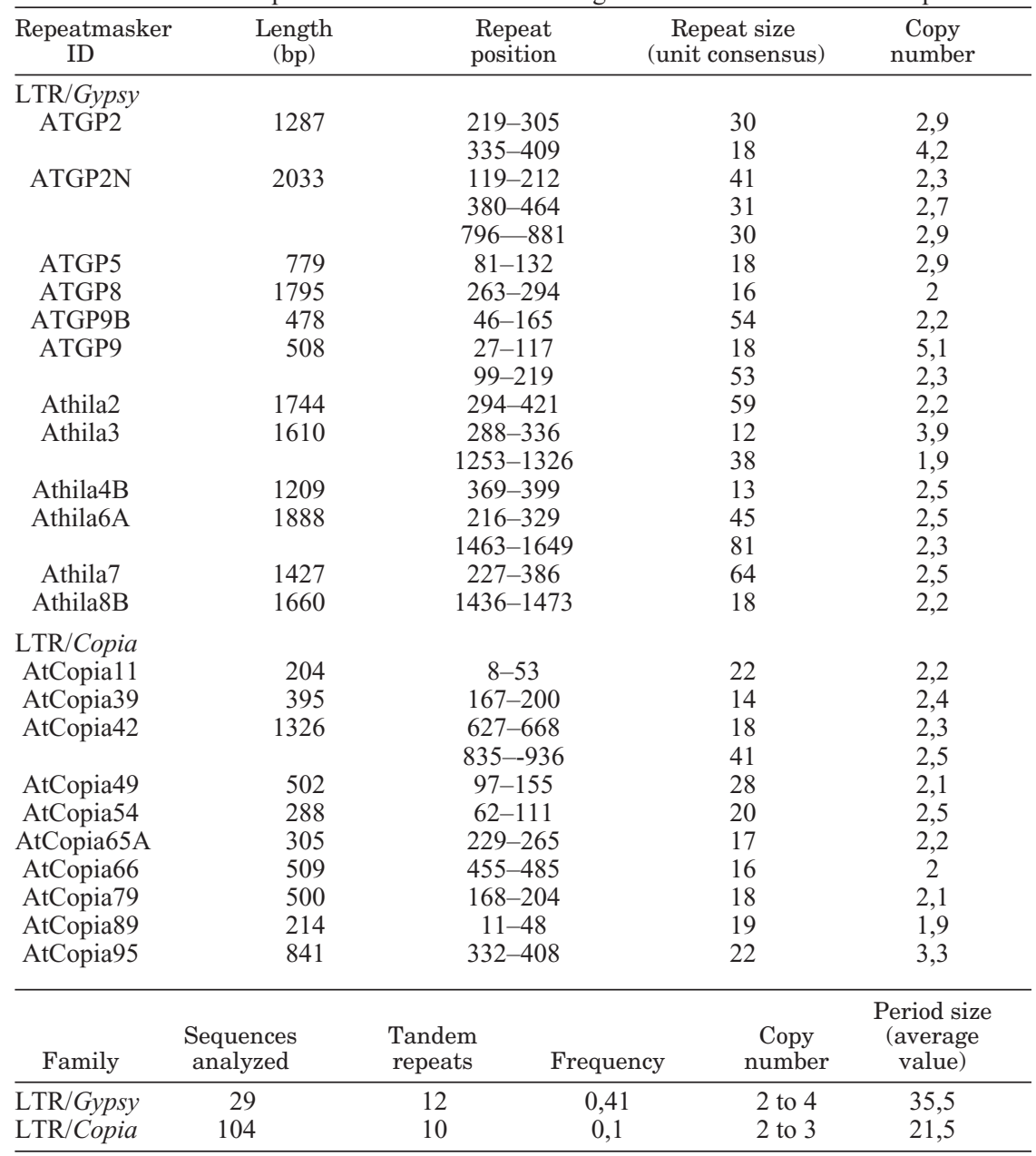

Sequences and nomenclature of retrotransposons were extracted from the Repeatmasker library (URL: www.girinst.org) and searched for tandem repeats with "tandem repeat finder" using default options (URL: tandem.bu.edu/trf/trf.html). Summaries for the Gypsy and Copia families are shown at the bottom.

to generate double-stranded RNA homologous to these promoter elements (here we use an intergenic solo LTR as an example). A double-stranded RNA could either be encoded by other copies of the LTR elsewhere in the genome and act in trans on the target LTR, or it could be generated in cis at the target locus. This can occur by pol II readthrough transcription from other transposon promoters or adjacent plant genes, with the overlapping transcripts annealing to form double-stranded RNA. Alternatively, pol II synthesis of a single-stranded primary transcript that is aberrant (e.g., lacking a poly(A) tail or $5^{\prime}$-cap structure) can be a template for RDR2, which would synthesize the second RNA strand. Following dicing of the double-stranded RNAs by DCL3, the resulting small RNAs can guide DRD1 and pol IVb to facilitate de novo methylation of the target LTR. This step might not require extensive transcription by the DRD1/pol $\mathrm{IVb}$ complex but simply the establishment of an open chromatin conformation at the small RNA-targeted site that exposes DNA in this region to DNA methyltransferases. The extended CTD of NRPD1b, including the 16-amino- acid repeats, might provide a platform for recruiting the DNA methyltransferase DRM2, which catalyzes de novo methylation of cytosines in all sequence contexts.

Once an LTR is methylated, pol II cannot initiate transcription from this promoter. However, pol IVa, which is proposed to be specialized for transcribing methylated DNA (Herr et al. 2005; Onodera et al. 2005), would be able to recognize and transcribe the methylated LTR. The LTR-containing transcript produced by pol IVa is then copied by RDR2 and diced by DCL3. The resulting small RNAs are again used by the DRD1/pol IVb machinery to maintain and even enhance methylation, which in turn would provoke increased transcription by pol IVa and elevated amounts of small RNAs, thus fueling the entire cycle. In this scenario, pol IVb-facilitated DNA methylation is restricted to regions targeted by small RNAs, whereas pol IVa transcription is confined to DNA sequences that are modified by methylation. The two pol IV complexes thus act in a concerted manner to both initiate and maintain DNA methylation that blocks pol II transcription from the target promoter. 


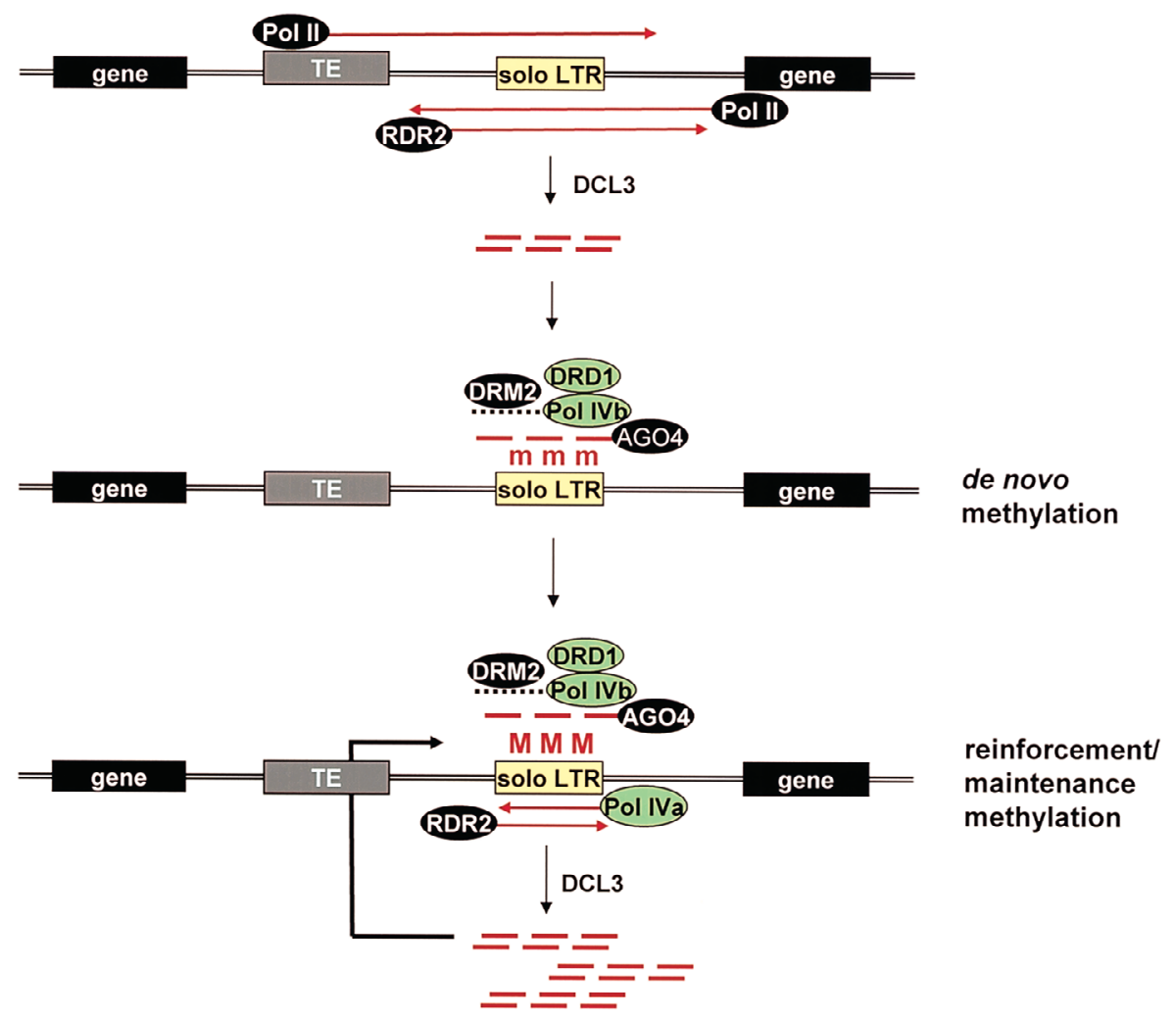

Figure 3. Hypothetical model for concerted action of pol IV complexes in RNA-directed de novo and maintenance methylation. The target is an intergenic solo LTR (yellow) located in euchromatin. (Red) RNAs. (Top) Double-stranded RNAs containing LTR sequences can potentially form by annealing of overlapping pol II transcripts initiating in adjacent transposon or plant gene promoters or by RDR2 activity on a single-stranded, aberrant pol II transcript. Double-stranded RNAs are processed to 24-nucleotide small RNAs by DCL3. (Middle) De novo methylation (small $\mathrm{m}$ ) in response to RNA signals is catalyzed primarily by DRM2, which we speculate can interact with the repeats in the CTD of NRPD1b, the largest subunit of pol IVb (short dotted black line). DRD1 is a putative SWI/SNF-like factor that might facilitate opening of chromatin at the small RNA-targeted site. AGO4 presumably interacts with small RNAs via its PAZ domain. (Bottom) pol IVa preferentially transcribes methylated DNA, producing aberrant transcripts that are copied by RDR2. The resulting double-stranded RNA is processed by DCL3, leading to reinforcement and maintenance of methylation (large $\mathrm{M})$, again by the pol IVb/DRD1/DRM2 complex. (Green) Components found only in plants.

\section{REVERSIBLE RNA-DIRECTED DNA METHYLATION IS HIGHLY DEVELOPED IN PLANTS}

Many components of the reversible RNA-directed DNA methylation machinery - including pol IVa, pol IVb, and DRD1 - as well as asymmetrical CNN methylation and ROS1 are found only in plants. Why do plants need this silencing pathway? One reason is to silence transposons that integrate into euchromatin and/or are too short to be stably packaged into constitutive heterochromatin (Tran et al. 2005). In addition, the DRD1/pol IVb pathway might benefit plants by contributing to a genome-wide system of small RNA-regulated, transposon-derived promoters/ enhancers that modulate gene expression under changeable and/or suboptimal growth conditions. Stress can induce the production of small RNAs (Sunkar and Zhu 2004) and reactivate retrotransposon LTRs to influence transcription of neighboring plant genes (Kashkush et al. 2003). These findings are consistent with a scenario in which LTRs and small RNAs are key participants in reversible DRD1/pol IVb-dependent silencing. There are more than 3000 annotated solo LTRs in the $A$. thaliana genome (Peterson-Burch et al. 2004), many of which reside in intergenic regions of euchromatic chromosome arms. These solo LTRs could supply epigenetically controlled rheostats or switches for nearby genes, whose increased or decreased expression might have adaptive significance for the plant. If so, the DRD1/pol IV pathway might fulfill a need for rapid, reversible changes in gene expression that help sessile plants to cope with environmental challenges.

\section{RNA-DIRECTED DNA METHYLATION IN HUMAN CELLS}

The possibility that RNA-directed DNA methylation occurs in human cells was first suggested by experiments in which small RNAs homologous to either transgene or endogenous gene promoters appeared to trigger transcriptional gene silencing and promoter methylation (Kawasaki and Taira 2004; Morris et al. 2004). The most recent studies suggest, however, that promoter-directed small RNAs induce either very low levels of methylation or no detectable methylation of target promoters in human cells (Castanotto et al. 2005; Ting et al. 2005). Nevertheless, promoter-directed small RNAs appear to 
reliably induce transcriptional gene silencing associated with $\mathrm{H} 3 \mathrm{~K} 9$ and H3K27 methylation in these cells (Ting et al. 2005; Weinberg et al. 2005). Given that crucial components of the RNA-directed DNA methylation machinery are found only in the plant kingdom, it is not too surprising that mammals do not have an identical mechanism for eliciting small RNA-directed transcriptional gene silencing and chromatin modifications.

Although the incidence and function of RNA-mediated transcriptional silencing in mammalian cells remain to be determined, there is considerable interest in the possibility that promoter-directed small RNAs might have therapeutic value in human medicine (Morris 2006). For instance, it has been reported that small RNAs directed to the LTR promoter region of human immunodeficiency virus type 1 (HIV-1) induce prolonged transcriptional gene silencing in cell culture (Suzuki et al. 2005). There are also possible disease connections. For example, even low levels of RNA-directed DNA methylation might have implications for cancer development if rare transcriptional silencing of a tumor suppressor gene confers a growth advantage (Castanotto et al. 2005).

\section{RNA POLYMERASE II INVOLVEMENT IN EPIGENETIC GENE SILENCING}

Even though pol IV appears to be specific to plants, there is evidence that another RNA polymerase - pol IIis required for RNAi-mediated heterochromatin formation in fission yeast. Genetic screens have identified roles in this process for three pol II subunits: Rpb7, Rpb2, and Rpb1. Interestingly, the subunits are needed at different steps of the pathway, which is reminiscent of the distinct contributions of the two pol IV complexes to RNAdirected DNA methylation in plants. In fission yeast, $\mathrm{Rpb} 7$ is required for synthesis of the precursor of small RNAs (Djupedal et al. 2005), whereas Rpb2 is needed to generate small RNAs from the precursor RNA (Kato et al. 2005). The largest pol II subunit Rpb1, in particular, the CTD, is needed downstream from these steps to convert small RNAs into chromatin modifications (Schramke et al. 2005). An involvement of pol II in RNAi-mediated transcriptional silencing in human cells is suggested by the sensitivity of this process to $\alpha$-amanitin (Weinberg et al. 2005). Thus, even though plants have evolved specialized pol IV complexes for RNA-directed DNA methylation, pol II appears to be able to carry out a related role in RNAi-mediated heterochromatin assembly in fission yeast and possibly humans. Presumably, pol IV complexes in plants are uniquely suited to dealing with the complexities of RNA-directed DNA methylation, as evidenced by their involvement in multiple stages of this process including not only de novo and maintenance methylation, but also possibly active demethylation.

\section{CONCLUSIONS}

The requirement for DNA-dependent RNA polymerases in gene silencing has been regarded as "paradoxical" (Vaughn and Martienssen 2005) and "counter-intuitive" (Huisinga et al. 2006). Yet the clear evidence for pol IV involvement in RNAi-mediated chromatin-based silencing in plants has confirmed that these multisubunit enzymes are key players in epigenetic regulation. The elaboration of RNA-directed DNA methylation and the pol IV pathway in the plant kingdom probably reflects the need for flexible mechanisms of gene regulation that enable swift responses to an ever-changing environment. The likelihood that dispersed transposon promoters are agents of this epigenetic regulatory system validates Barbara McClintock's concept of transposons as "controlling elements" (Shapiro 2005). That the functions of pol IV complexes were first discerned in genetic analyses of transgene silencing attests to the fruitfulness of studying "foreign" DNA in plants (Matzke and Matzke 2004). Future work will address the many aspects of pol IV that remain to be clarified, including the mechanisms by which the two pol IV complexes act at discrete steps of the RNA-directed DNA methylation pathway, the complete catalog of target sequences, and the full range of functions in plant physiology and development.

\section{ACKNOWLEDGMENTS}

Financial support has been provided by the Austrian Fonds zur Förderung der wissenschaftlichen Forschung (grant numbers I26-B03 and P-16545-B12) and the European Union (contract number HPRN-CT 2002-00257).

\section{REFERENCES}

Arabidopsis Genome Initiative. 2000. Analysis of the genome sequence of the flowering plant Arabidopsis thaliana. Nature 408: 796.

Aufsatz W., Mette M.F., Matzke A.J.M., and Matzke M. 2004. The role of MET1 in RNA-directed de novo and maintenance methylation of CG dinucleotides. Plant Mol. Biol. 54: 793.

Aufsatz W., Mette M.F., van der Winden J., Matzke A.J.M., and Matzke M.A. 2002a. RNA-directed DNA methylation in Arabidopsis. Proc. Natl. Acad. Sci. 99: 16499.

Aufsatz W., Mette M.F., van der Winden J., Matzke M., and Matzke A.J.M. 2002b. HDA6, a putative histone deacetylase needed to enhance DNA methylation induced by double stranded RNA. EMBO J. 21: 6832 .

Bellaoui M. and Gruissem W. 2004. Altered expression of the Arabidopsis ortholog of DCL affects normal plant development. Planta 219: 819.

Bernstein E. and Allis C.D. 2005. RNA meets chromatin. Genes Dev. 19: 1635 .

Buratowski S. 2003. The CTD code. Nat. Struct. Biol. 10: 679.

Cam H.P., Sugiyama T., Chen E.S., Chen X., FitzGerald P.C., and Grewal S.I. 2005. Comprehensive analysis of heterochromatin- and RNAi-mediated epigenetic control of the fission yeast genome. Nat. Genet. 37: 809 .

Cao X., Springer N.M., Muszynski M.G., Phillips R.L., Kaeppler S., and Jacobsen S.E. 2000. Conserved plant genes with similarity to mammalian de novo DNA methyltransferases. Proc. Natl. Acad. Sci. 97: 4979.

Cao X., Aufsatz W., Zilberman D., Mette M.F., Huang M.S., Matzke M., and Jacobsen S.E. 2003. Role of the DRM and CMT3 methyltransferases in RNA-directed DNA methylation. Curr. Biol. 13: 2212.

Carty S.M. and Greenleaf A.L. 2002. Hypophosphorylated Cterminal repeat domain-associating proteins in the nuclear proteome link transcription to DNA/chromatin modification and RNA processing. Mol. Cell. Proteomics 1: 598.

Castanotto D., Tommasi S., Li M., Li H., Yanow S., Pfeifer G.P., and Rossi J.J. 2005. Short hairpin RNA-directed cytosine (CpG) methylation of the RASSF $1 A$ gene promoter in HeLa cells. Mol. Ther. 12: 179. 
Chan S.W.-L., Henderson I.R., and Jacobsen S.E. 2005. Gardening the genome: DNA methylation in Arabidopsis thaliana. Nat. Rev. Genet. 6: 351.

Dalmay T., Hamilton A., Rudd S., Angell S., and Baulcombe D.C. 2000. An RNA-dependent RNA polymerase gene in Arabidopsis is required for posttranscriptional gene silencing mediated by a transgene but not by a virus. Cell 101: 543.

Dennis K., Fan T., Geiman T., Yan Q., and Muegge K. 2001. Lsh, a member of the SNF2 family, is required for genomewide methylation. Genes Dev. 15: 2940.

Djupedal I., Portoso M., Spahr H., Bonilla C., Gustafsson C.M., Allshire R.C., and Ekwall K. 2005. RNA Pol II subunit Rpb7 promotes centromeric transcription and RNAi-directed chromatin silencing. Genes Dev. 19: 2301.

Fuchs J., Demidov D., Houben A., and Schubert I. 2006. Chromosomal histone modification patterns-From conservation to diversity. Trends Plant Sci. 11: 199.

Hampsey M. and Reinberg D. 2003. Tails of intrigue: Phosphorylation of RNA polymerase II mediates histone methylation. Cell 113: 429.

Herr A.J. 2005. Pathways through the small RNA world of plants. FEBS Lett. 579: 5879 .

Herr A.J., Jensen M.B., Dalmay T., and Baulcombe D.C. 2005. RNA polymerase IV directs silencing of endogenous DNA. Science 308: 118.

Huettel B., Kanno T., Daxinger L., Aufsatz W., Matzke A.J.M., and Matzke M. 2006. Endogenous targets of RNA-directed DNA methylation and Pol IV in Arabidopsis. EMBO J. 25: 2828.

Huisinga K.L., Brower-Toland B., and Elgin S.C.R. 2006. The contradictory definitions of heterochromatin: Transcription and silencing. Chromosoma 115: 110.

Jackson J.P., Lindroth A.M., Cao X., and Jacobsen S.E. 2002. Control of CpNpG DNA methylation by the KRYPTONITE histone H3 methyltransferase. Nature 416: 556.

Jeddeloh J.A., Stokes T.L., and Richards E.J. 1999. Maintenance of genomic methylation requires a SWI2/SNF2-like protein. Nat. Genet. 22: 94.

Jones L., Ratcliff F., and Baulcombe D.C. 2001. RNA-directed transcriptional gene silencing in plants can be inherited independently of the RNA trigger and requires Met1 for maintenance. Curr. Biol. 11: 747.

Jones L., Hamilton A.J., Voinnet O., Thomas C.L., Maule A.J., and Baulcombe D.C. 1999. RNA-DNA interactions and DNA methylation in post-transcriptional gene silencing. Plant Cell 11: 2291

Kanno T., Aufsatz W., Jaligot E., Mette M.F., Matzke M., and Matzke A.J.M. 2005a. A SNF2-like protein facilitates dynamic control of DNA methylation. EMBO Rep. 6: 649.

Kanno T., Mette M.F., Kreil D.P., Aufsatz W., Matzke M., and Matzke A.J.M. 2004. Involvement of putative SNF2 chromatin remodelling protein DRD1 in RNA-directed DNA methylation. Curr. Biol. 14: 801.

Kanno T., Huettel B., Mette M.F., Aufsatz W., Jaligot E., Daxinger L., Kreil D.P., Matzke M., and Matzke A.J.M. 2005b. Atypical RNA polymerase subunits required for RNA-directed DNA methylation. Nat. Genet. 37: 761.

Kapoor A., Agius F., and Zhu J.K. 2005. Preventing transcriptional gene silencing by active DNA demethylation. FEBS Lett. 579: 5889.

Kashkush K., Feldman M., and Levy A.A. 2003. Transcriptional activation of retrotransposons alters the expression of adjacent genes in wheat. Nat. Genet. 33: 102.

Kato H., Goto D.B., Martienssen R.A., Urano T., Furukawa K., and Murakami Y. 2005. RNA polymerase II is required for RNAi-dependent heterochromatin assembly. Science 309: 467.

Kavi H.H., Xie W., Fernandez H.R., and Birchler J.A. 2005. Global analysis of siRNA-mediated transcriptional gene silencing. BioEssays 27: 1209.

Kawasaki H. and Taira K. 2004. Induction of DNA methylation and gene silencing by short interfering RNAs in human cells. Nature 431: 211.
Lahmy S., Guilleminot J., Cheng C.-M., Bechtold N., Albert S., Pelletier G., Delseny M., and Devic M. 2004. DOMINO1, a member of a small plant-specific gene family, encodes a protein essential for nuclear and nucleolar functions. Plant J. 39: 809.

Lu C., Tej S.S., Luo S., Haudenschild C.D., Meyers B.C., and Green P.J. 2005. Elucidation of the small RNA component of the transcriptome. Science 309: 467.

Malagnac F., Bartee L., and Bender J. 2002. An Arabidopsis SET domain protein required for maintenance but not establishment of DNA methylation. EMBO J. 21: 6842.

Martienssen R.A. 2003. Maintenance of heterochromatin by RNA interference of tandem repeats. Nat. Genet. 35: 213.

Mathieu O. and Bender J. 2004. RNA-directed DNA methylation. J. Cell Sci. 117: 4861.

Matzke M.A. and Birchler J.A. 2005. RNAi-mediated pathways in the nucleus. Nat. Rev. Genet. 6: 24.

Matzke M.A. and Matzke A.J.M. 2004. Planting the seeds of a new paradigm. PLoS Biol. 2: E133.

Matzke M.A., Primig M., Trnovsky J., and Matzke A.J.M. 1989. Reversible methylation and inactivation of marker genes in sequentially transformed plants. EMBO J. 8: 643.

Meinhart A., Kamenski T., Hoeppner S., Baumli S., and Cramer P. 2005. A structural perspective of CTD function. Genes Dev. 19: 1401 .

Mette M.F., van der Winden J., Matzke M.A., and Matzke A.J.M. 1999. Production of aberrant promoter transcripts contributes to methylation and silencing of unlinked homologous promoters in trans. EMBO J. 18: 241.

Mette M.F., Aufsatz W., van der Winden J., Matzke M.A., and Matzke A.J.M. 2000. Transcriptional silencing and promoter methylation triggered by double stranded RNA. EMBO J. 19: 5194.

Morales-Ruiz T., Ortega-Galisteo A.P., Ponferrada-Marin M.I., Martinez-Macias M.I., Ariza R.R., and Roldán-Aroja T. 2006. DEMETER and REPRESSOR OF SILENCING 1 encode 5-methylcytosine DNA glycosylases. Proc. Natl. Acad. Sci. 103: 6853.

Morris K.V. 2006. Therapeutic potential of siRNA-mediated transcriptional gene silencing. Biotechniques (suppl.) 2006: 7.

Morris K.V., Chan S.W., Jacobsen S.E., and Looney D.J. 2004. Small interfering RNA-induced transcriptional gene silencing in human cells. Science 305: 1289.

Onodera Y., Haag J.R., Ream T., Costa Nunes P., Pontes O., and Pikaard C.S. 2005. Plant nuclear RNA polymerase IV mediates siRNA and DNA methylation-dependent heterochromatin formation. Cell 120: 613.

Park Y.D., Papp I., Moscone E.A., Iglesias V.A., Vaucheret H., Matzke A.J.M, and Matzke M.A. 1996. Gene silencing mediated by promoter homology occurs at the level of transcription and results in meiotically heritable alterations in methylation and gene activity. Plant J. 9: 183 .

Pélissier T. and Wassenegger M. 2000. A DNA target of $30 \mathrm{bp}$ is sufficient for RNA-directed DNA methylation. RNA 6: 55 .

Pélissier T., Thalmeir S., Kempe D., Sänger H.-L., and Wassenegger M. 1999. Heavy de novo methylation at symmetrical and non-symmetrical sites is a hallmark of RNAdirected DNA methylation. Nucleic Acids Res. 27: 1625.

Peterson-Burch B.D., Nettleton D., and Voytas D.F. 2004. Genomic neighbourhoods for Arabidopsis retrotransposons: A role for targeted integration in the distribution of the Metaviridae. Genome Biol. 5: R78.

Pontier D., Yahubyan G., Vega D., Bulski A., Saez-Vasquez J., Hakimi M.A., Lerbs-Mache S., Colot V., and Lagrange T. 2005. Reinforcement of silencing at transposons and highly repeated sequences requires the concerted action of two distinct RNA polymerases IV in Arabidopsis. Genes Dev. 19: 2030.

Schramke V. and Allshire R. 2003. Hairpin RNAs and retrotransposon LTRs effect RNAi and chromatin-based gene silencing. Science 301: 1069.

Schramke V., Sheedy D.M., Denli A.M., Bonila C., Ekwall K., Hannon G.J., and Allshire R.C. 2005. RNA-interferencedirected chromatin modification coupled to RNA polymerase II transcription. Nature 435: 1275. 
Shapiro J.A. 2005. Retrotransposons and regulatory suites BioEssays 27: 122.

Sijen T., Vijn I., Rebocho A., van Blokland R., Roelofs D., Mol J.N.M., and Kooter J.M. 2001. Transcriptional and posttranscriptional gene silencing are mechanistically related. Curr. Biol. 11: 436.

Sunkar R. and Zhu J.K. 2004. Novel and stress-regulated microRNAs and other small RNAs from Arabidopsis. Plant Cell 16: 2001.

Suzuki K., Shijuuku T., Fukamachi T., Zaunders J., Guillemin G., Cooper D., and Kelleher A. 2005. Prolonged transcriptional silencing and $\mathrm{CpG}$ methylation induced by siRNAs targeted to the HIV-1 promoter region. J. RNAi Gene Silencing 1: 66 .

Ting A.H., Schuebel K.E., Herman J.G., and Baylin S.B. 2005. Short double-stranded RNA induces transcriptional gene silencing in human cancer cells in the absence of DNA methylation. Nat. Genet. 37: 906.

Tran R., Zilberman D., de Bustos C., Ditt R.F., Henikoff J.G., Lindroth A.M., Delrow J., Boyle T., Kwong S., Bryson T.D., et al. 2005. Chromatin and siRNA pathways cooperate to maintain DNA methylation of small transposable elements in
Arabidopsis. Genome Biol. 6: R90.

Vaucheret H. 1993. Identification of a general silencer for $19 \mathrm{~S}$ and $35 \mathrm{~S}$ promoters in a transgenic tobacco plant: $90 \mathrm{bp}$ of homology in the promoter sequence are sufficient for transinactivation. C.R. Acad. Sci. 316: 1471.

. 2005. RNA polymerase IV and transcriptional silencing. Nat. Genet. 37: 659.

Vaughn M.W. and Martienssen R.A. 2005. Finding the right template: RNA Pol IV, a plant-specific RNA polymerase. Mol. Cell 17: 754.

Wassenegger M., Heimes S., Riedel L., and Sänger H.-L. 1994. RNA-directed de novo methylation of genomic sequences in plants. Cell 76: 567.

Weinberg M.S., Villeneuve L.M., Ehsani A., Aagaard L., Chen Z.X., Riggs A.D., Rossi J.J., and Morris K.V. 2005. The antisense strand of small interfering RNAs directs histone methylation and transcriptional gene silencing in human cells. $R N A$ 12: 256 .

Xie Z., Johansen L.K., Gustafson A.M., Kasschau K.D., Lellis A.D., Zilberman D., Jacobsen S.E., and Carrington J.C. 2004. Genetic and functional diversification of small RNA pathways in plants. PLoS Biol. 2: E104. 


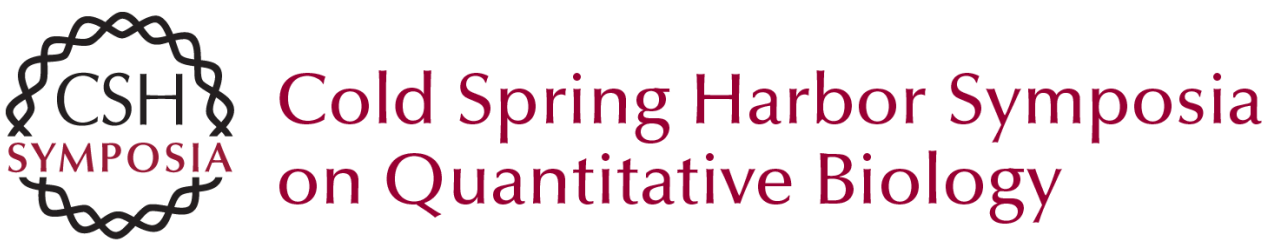

\section{RNA-directed DNA Methylation and Pol IVb in Arabidopsis}

M. MATZKE, T. KANNO, B. HUETTEL, et al.

Cold Spring Harb Symp Quant Biol 2006 71: 449-459

Access the most recent version at doi:10.1101/sqb.2006.71.028

References This article cites 68 articles, 22 of which can be accessed free at: http://symposium.cshlp.org/content/71/449.full.html\#ref-list-1

\section{License}

Email Alerting Receive free email alerts when new articles cite this article - sign up in Service the box at the top right corner of the article or click here. 